\title{
Development of Commercially Viable Compliant Mechanisms Using the Pseudo-Rigid-Body Model: Case Studies of Parallel Mechanisms
}

Christopher A. Mattson

Larry L. Howell

Ihowell@byu.edu

Spencer P. Magleby

magleby@byu.edu

Follow this and additional works at: https://scholarsarchive.byu.edu/facpub

Part of the Mechanical Engineering Commons

\section{Original Publication Citation}

Mattson, C.A., Howell, L.L., and Magleby, S.P., Development of Commercially Viable Compliant Mechanisms Using the Pseudo-Rigid-Body Model: Case Studies of Parallel Mechanisms, Journal of Intelligent Material Systems and Structures, Vol. 15, No. 3, pp. 195-22, 24.

\section{BYU ScholarsArchive Citation}

Mattson, Christopher A.; Howell, Larry L.; and Magleby, Spencer P., "Development of Commercially Viable Compliant Mechanisms Using the Pseudo-Rigid-Body Model: Case Studies of Parallel Mechanisms" (2004). Faculty Publications. 451.

https://scholarsarchive.byu.edu/facpub/451

This Peer-Reviewed Article is brought to you for free and open access by BYU ScholarsArchive. It has been accepted for inclusion in Faculty Publications by an authorized administrator of BYU ScholarsArchive. For more information, please contact ellen_amatangelo@byu.edu. 


\section{DEVELOPMENT OF COMMERCIALLY VIABLE COMPLIANT MECHANISMS USING THE PSEUDO-RIGID-BODY MODEL: CASE STUDIES OF PARALLEL MECHANISMS}

\author{
Christopher A. Mattson \\ Mechanical, Aerospace, and Nuclear Eng. \\ Rensselaer Polytechnic Institute \\ Troy, New York, 12180 \\ mattsc@rpi.edu
}

\author{
Larry L. Howell \\ Department of Mechanical Engineering \\ Brigham Young University \\ Provo, Utah, 84602 \\ lhowell@et.byu.edu
}

\author{
Spencer P. Magleby \\ Department of Mechanical Engineering \\ Brigham Young University \\ Provo, Utah, 84602 \\ magleby@byu.edu
}

\begin{abstract}
Analysis and synthesis of compliant mechanisms has recently been the subject of significant study in the research community. This focus has led to a number of design approaches for developing compliant mechanisms. This paper describes the value of using the Pseudo-Rigid-Body Model (PRBM) to design compliant mechanisms for commercial products. Application of the PRBM is illustrated through the development of two parallel mechanisms: a bicycle derailleur and parallel-motion bicycle brakes. The PRBM allows compliant mechanisms to be modeled and analyzed as rigid-body mechanisms and significantly reduces the complexity of analysis. Mechanisms with straightforward properties are used to demonstrate the use of the PRBM to design commercially viable compliant mechanisms for required motion and force-deflection characteristics.
\end{abstract}

Keywords: Compliant Mechanisms, Pseudo-Rigid-Body Model, Parallel Mechanisms, Bicycle Derailleur, Bicycle Brakes

\section{INTRODUCTION}

Analysis and synthesis of compliant mechanisms has been the subject of significant study in the research community (Ananthasuresh and Kota, 1995, Pedersen et al., 1999, Frecker, 2000). This focus has led to a number of design approaches for developing compliant mechanisms. These approaches have largely been applied in laboratory-like settings, and are just now seeing some application to commercially-viable products (Berglund et al., 2000).

The ability to easily evaluate the motion and force-deflection characteristics for a particular configuration is a critical element in designing compliant mechanisms for product applications. The objective of this paper is to describe an approach to using the Pseudo-Rigid-Body Model (PRBM) to determine these characteristics as part of the design of compliant mechanisms for commercial products. Application of the PRBM is illustrated through the development of two products: a bicycle derailleur and parallel-motion bicycle brakes.

Both case studies are parallel mechanisms, having opposing links that remain parallel throughout the motion of the mechanism. Compliant parallel mechanisms provide this motion through the deflection of flexible members. Appropriately designed, they can have characteristics of high precision, repeatability, integrated springs, low weight, and reduced wear and part count (Derderian et al., 1996). In many applications (including the two described in this paper), these characteristics can make compliant parallel mechanisms more attractive than their rigid-body counterparts.

To achieve the range of motion commonly associated with parallel-mechanism applications, the flexible members of a compliant parallel-mechanism must undergo large, nonlinear deflections. The PRBM allows such mechanisms to be modeled and analyzed as rigid-body mechanisms and significantly reduces the complexity of analysis. This is particularly useful in the early phases of design where appropriate geometry must be determined to assure that a mechanism will achieve the required motion. The analysis results of the paper are supported by the fabrication and testing of the case study prototypes, and the demonstration of successful mass production of the bicycle brakes.

The paper first presents a brief introduction to compliant mechanisms, the PRBM and parallel mechanisms. This is followed by the presentation of two case studies: the compliant bicycle derailleur and compliant parallel-motion bicycle brakes. Finally, the paper ends with conclusions that can be drawn from this study.

\section{BACKGROUND}

The two case studies presented in this paper are compliant mechanisms that gain some of their motion from the deflection of flexible members. Compliant mechanisms offer many potential advantages in product design such as reduction of weight, part count, backlash and joint wear, and decreased cost due to simplified manufacturing and assembly. Other advantages include high precision, and potential energy storage in deflected members. Some potential disadvantages associated with compliant mechanisms are difficulty of analysis, and high dependence on material properties for determining functionality (Howell et. al., 1996). 


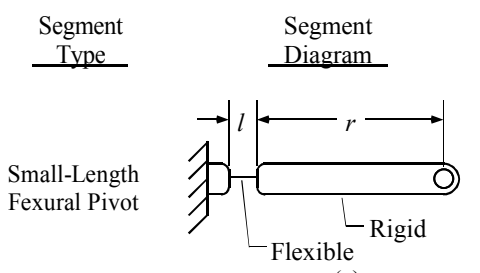

(a)

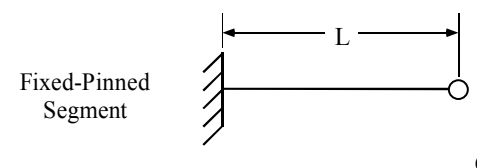

(c)

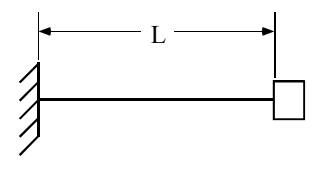

(e)

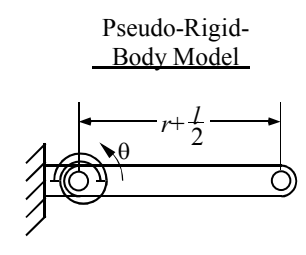

(b)

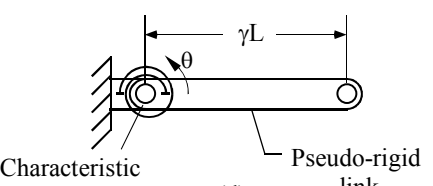

(d)

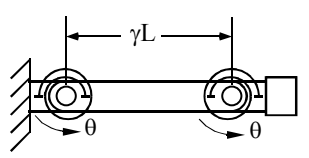

(f)
Figure 1. Typical compliant mechanism segments and their PRBM representation

The compliant bicycle derailleur and brakes, as well as many other compliant mechanisms, experience large nonlinear deflections. The PRBM provides a simple method for analyzing compliant members that undergo nonlinear deflections by modeling their deflection using rigid-body components that have equivalent force-deflection characteristics. Torsional springs are added to model stiffness and potential energy. In addition to the relatively simple motion of the parallel mechanism case studies presented here, the PRBM is capable of anlyzing complex motion.

Figure 1 shows typical segments found in compliant mechanisms and their pseudo-rigid-body representations. In addition, type synthesis is used to identify mehcnaism configurations throughout the design space, thus facilitating broad protection of intellectual property.

Figure 2 illustrates the motion of a rigid-link parallel mechanism. The mechanism is a simple four-bar in which the opposing links have the same lengths, thus forming a parallelogram. Rigid-link parallel mechanisms have found use in a variety of practical applications, including high-speed train catenaries, positioning of optics, amusement park rides, and consumer products such as desk lamps and fishing tackle boxes.

Compliant parallel mechanisms can be designed so that they retain all the advantages associated with rigid-body parallel mechanisms, including those discussed above. A compliant parallel-mechanism is shown in Figure 3. The mechanisms in Figure 2 and Figure 3 have the same motion.

In the case studies that follow, the use of the PRBM is emphasized as it was critical to performing the following design activities:
1. Identify, analyze, and select an appropriate compliant configuration

2. Calculate the force-deflection relationships

3. Perform stress and fatigue analyses

4. Select materials appropriate for the given stress and fatigue loads, considering necessary force constraints

5. Determine dimensions that meet constraints of fatigue life and return force

\section{CASE STUDY - COMPLIANT BICYCLE DERAILLEUR}

Recent developments in the bicycle industry have focused on increased performance and decreased bicycle weight. These criteria have led to innovative designs and the use of advanced materials. This case study features a bicycle derailleur that was developed to increase bicycle performance and decrease weight using compliant mechanisms technology.

Twenty-eight possible design configurations for the compliant derailleur were generated using the PRBM and type synthesis (Derderian et al., 1996, Murphy et al., 1996). The method of type synthesis provides a means to determine mechanism topologies without consideration for the actual dimensions of links and segments. This identification of the design space provides more design options and is used in broadening intellectual property protection.

The configurations were evaluated to determine which would best undergo the desired motion while not violating constraints on stress, fatigue life, weight, size, and other design criteria. Analyses were performed on a number of configurations that showed the most promise. These configurations were further prototyped and tested, resulting in a derailleur with equal or better performance to traditional derailleurs and a 25 gram reduction in weight compared to a popular commercial model. This weight reduction is significant for a single component in high performance bicycles and can provide manufacturers with a competitive advantage. In addition, the final design concept could be implemented by various derailleur manufacturers with minimal changes to existing derailleur tooling. The discussion that follows is focused on the PRBM as a fundamental tool used to quickly and effectively design a marketable compliant bicycle derailleur.

First, a benchmark derailleur was chosen (Shimano Deore XT) and the rigid-body diagram, shown in Figure 4a, was extracted. The benchmark, as well as most rear bicycle derailleurs, is constructed using a parallel four-bar mechanism. These mechanisms are used to position a bicycle chain on the desired sprocket, while keeping the chain-guide parallel to the sprockets.

The compliant parallel-mechanism configuration chosen for the derailleur design was a four-bar mechanism with three rigid links, one compliant member, and two pin joints as shown in Figure $4 \mathrm{~b}$. The compliant member in this configuration is 


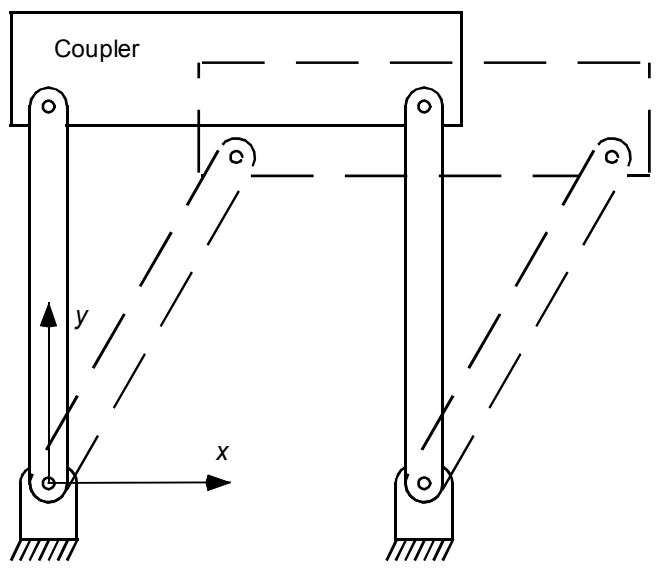

Figure 2. Parallel mechanism with rigid links
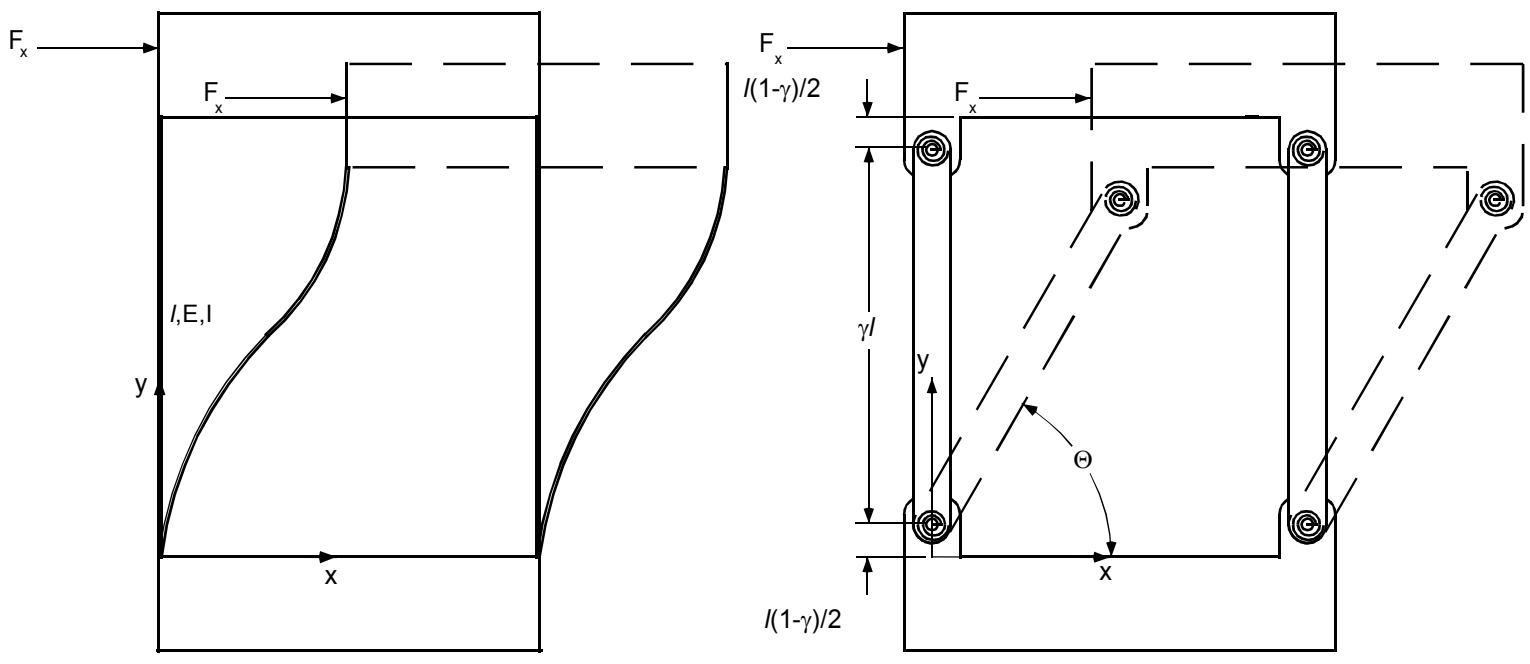

Figure 3. Compliant parallel mechanism and PRBM

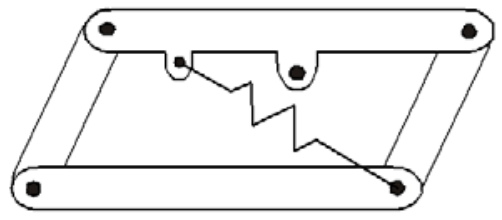

(a)

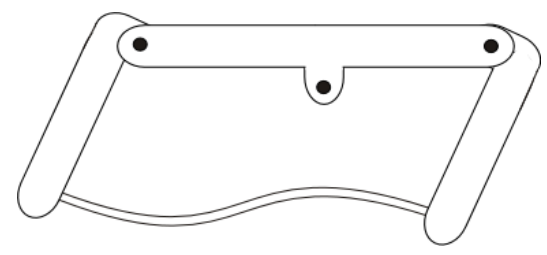

(b)

Figure 4. Rigid-body diagram (a) and compliant concept diagram (b) for the bicycle derailleur

constrained to a fixed-guided motion, where one of its ends is fixed and the other is constrained to have no rotational motion, but can be displaced in both the $x$ and $y$ direction. A diagram of the fixed-guided motion using a compliant beam and its corresponding PRBM is shown in Figure 5.
The PRBM for the compliant derailleur is created by substituting a pseudo-rigid-body element from Figure 5 for the compliant element as shown in Figure 4(b). The resulting PRBM diagram is shown in Figure 6. Further examination of the 

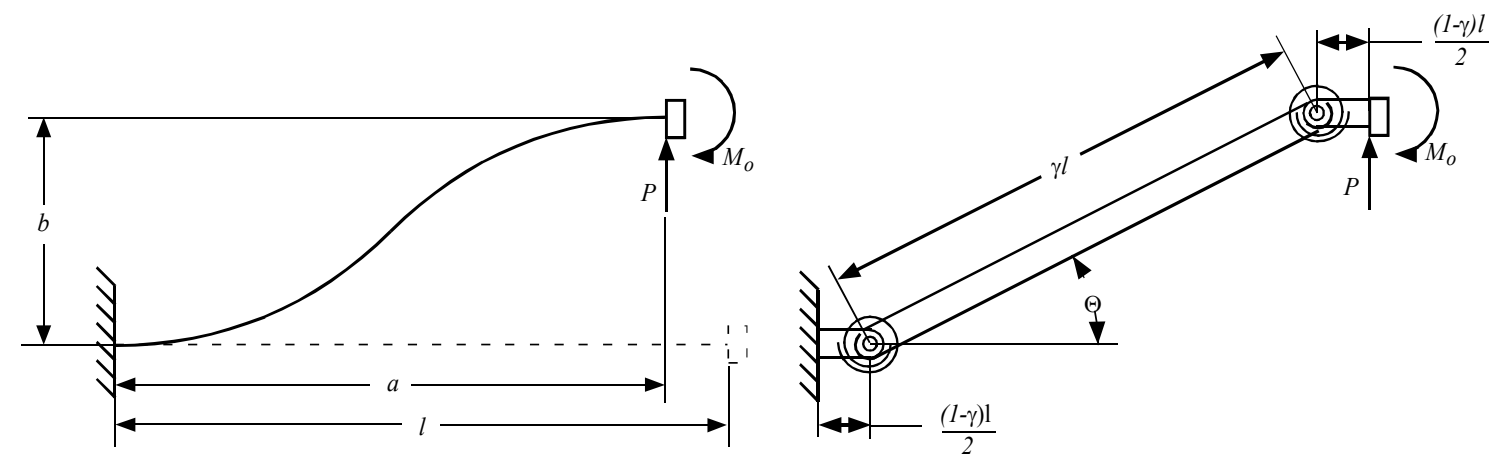

Figure 5. Fixed-Guided Motion; compliant mechanism (left) and corresponding PRBM (right)

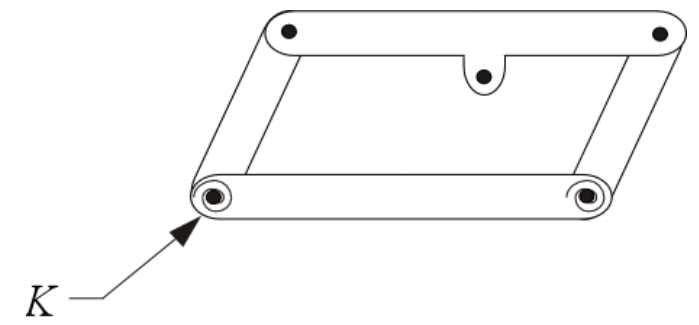

Figure 6. Pseudo-rigid-body model for the compliant derailleur

benchmark led to target performance values and geometric constraints, such as link lengths and mechanism deflection. These parameters and values help to further define the PRBM and are listed in Table 1.

Position, force-displacement, and stress relationships are calculated from the PRBM equations. Figure 5 shows the physical meaning of the variables. The position equations are

$$
a=l(1-\gamma(1-\cos (\Theta)))
$$

$$
b=\gamma l \sin (\Theta)
$$

where $\gamma$ is the characteristic radius factor, a nondimensional parameter used to define the location of the characteristic pivots and torsional springs. A value of 0.85 is appropriate for this case and the spring constant, $K$, is calculated by

$$
K=\frac{2 \gamma K_{\Theta} E I}{l}
$$

where $K_{\Theta}$ is approximated to be 2.7 (Howell, 2001). The maximum stress occurs at the ends of the beam and is

$$
S_{\max }=\frac{P a c}{2 I}
$$

where $c$ is the distance from the neutral axis to the outer fibers.

Considering the mechanism at its largest deflection $\left(b_{\max }=\right.$ $0.0432 \mathrm{~m}(1.70 \mathrm{in})$ ), which is the total width of the sprockets plus a preload, the rest of the values were calculated. From Equation (1), $a$ at $b_{\text {max }}$ is equal to $0.0185 \mathrm{~m}(0.73 \mathrm{in})$.

Table 1: Derailleur Design Parameters

\begin{tabular}{ccc}
\hline Parameter & Benchmark Value & Design Target Value \\
\hline Maximum Design Deflection & $0.0442 \mathrm{~m}(1.74 \mathrm{in})$ & $0.0432 \mathrm{~m}(1.70 \mathrm{in})$ \\
Maximum Target Deflection & $0.0381 \mathrm{~m}(1.5 \mathrm{in})$ & $0.0381 \mathrm{~m}(1.5 \mathrm{in})$ \\
Minimum Force at Maximum Deflection & $22.24 \mathrm{~N}(5 \mathrm{lbf})$ & $22.24 \mathrm{~N}(5 \mathrm{lbf})$ \\
Spring Preload & $4.45 \mathrm{~N}(1 \mathrm{lbf})$ & $4.45 \mathrm{~N}(1 \mathrm{lbf})$ \\
Longest Link Length & $0.0445 \mathrm{~m}(1.75 \mathrm{in})$ & $0.0445 \mathrm{~m}(1.75 \mathrm{in})$ \\
Cycle Life & $>100,000 \mathrm{cycles}$ & $>110,000 \mathrm{cycles}$ \\
Target Link Width & $0.0178 \mathrm{~m}(0.7 \mathrm{in})$ & $0.0157 \mathrm{~m}(0.617 \mathrm{in})$ \\
\hline
\end{tabular}


In general, materials with large ratios of yield strength $\left(S_{y}\right)$ to Young's modulus $(E)$ or $S_{y} / E$ are optimal materials for compliant mechanisms, as they are relatively strong and flexible. E-Glass, a type of fiberglass, has a large $\left(S_{y} / E\right)$ ratio and was chosen as the material for the compliant member. Material properties for two types of E-Glass were collected through tensile and bend tests, and the results are listed in Table 2 and Table 3. Layer configurations are described by stating the E-Glass configuration used for each layer. Under the "Value" column of the tables, the notation " $w$ " represents woven layers and " $o$ " represents a layer of unidirectional fibers aligned with the long axis of the compliant member. E-Glass Material 1 and 2 are fabricated of 4 and 5 layers respectively, each having woven material as the outermost layers of the composite.

With material properties known, Equation (3) yields $K=0.17$ $\mathrm{N}-\mathrm{m}$ (1.5 in-lbf) and Equation (4), yields $S_{\max }=1.1 \mathrm{GPa}$ $(150,000 \mathrm{psi})$. The bending stress safety factor for static failure at $b_{\max }$ and $S_{\max }$ for the beam is 1.72 . Using the PRBM, the force $(P)$ required to deflect the beam to various points from $b=0$ to $b_{\max }$, is calculated as

$$
P=\frac{4 K \Theta}{\gamma l \cos \Theta}
$$

Figure 7 plots the displacement path based on the PRBM equations and compares them to measured values of the displacement path for a prototype mechanism that was built and tested. The force and displacement were measured by fixing the longest rigid link of the mechanism, and using a linear actuator to create a displacement in the " $b$ " direction (see Figure 5). The

Table 2: E-Glass Material 1 Properties

\begin{tabular}{cc}
\hline Material Properties & Value \\
\hline Layer Configuration & w,o,o,w \\
Ultimate Tensile Strength $\left(\mathrm{S}_{\mathrm{ut}}\right)$ & $3.4 \mathrm{GPa}(500,000 \mathrm{psi})$ \\
Yield Strength $\left(\mathrm{S}_{\mathrm{y}}\right)$ & $1.8 \mathrm{GPa}(260,000 \mathrm{psi})$ \\
Young's Modulus $(\mathrm{E})$ & $9.9 \mathrm{GPa}(1,430,000 \mathrm{psi})$ \\
Poisson's Ratio $(\mathrm{v})$ & 0.30 \\
\hline
\end{tabular}

Table 3: E-Glass Material 2 Properties

\begin{tabular}{cc}
\hline Material Properties & Value \\
\hline Layer Configuration & w,o,o,o,w \\
Ultimate Tensile Strength $\left(\mathrm{S}_{\mathrm{ut}}\right)$ & $3.4 \mathrm{GPa}(500,000 \mathrm{psi})$ \\
Yield Strength $\left(\mathrm{S}_{\mathrm{y}}\right)$ & $3.8 \mathrm{GPa}(550,000 \mathrm{psi})$ \\
Young's Modulus $(\mathrm{E})$ & $15.6 \mathrm{GPa}(2,260,000 \mathrm{psi})$ \\
Poisson's Ratio $(\mathrm{v})$ & 0.30 \\
\hline
\end{tabular}

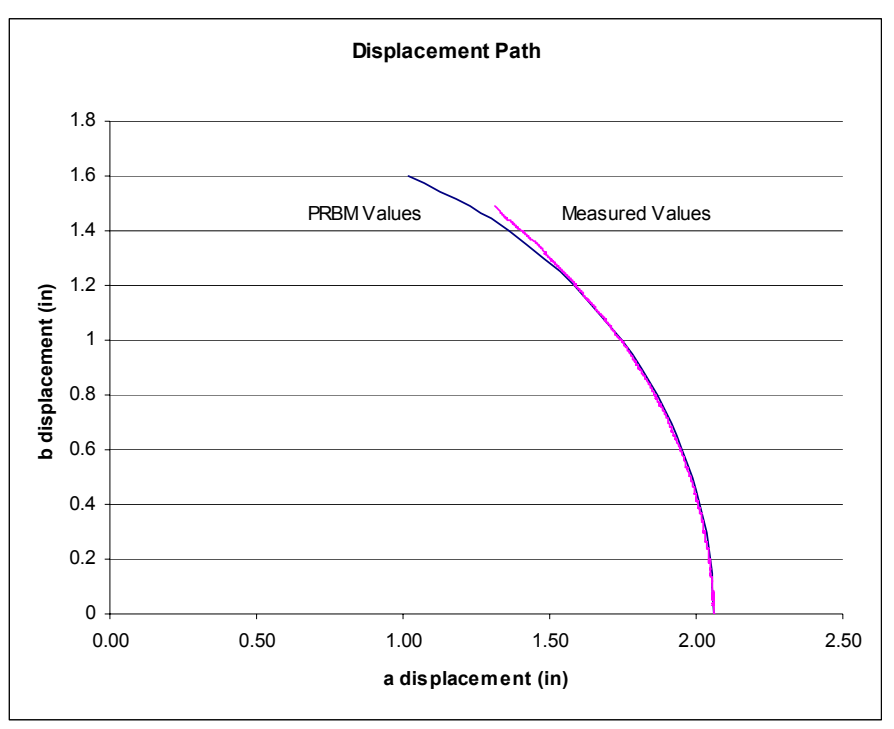

Figure 7. Measured values vs. PRBM calculated values of displacement of end of compliant beam

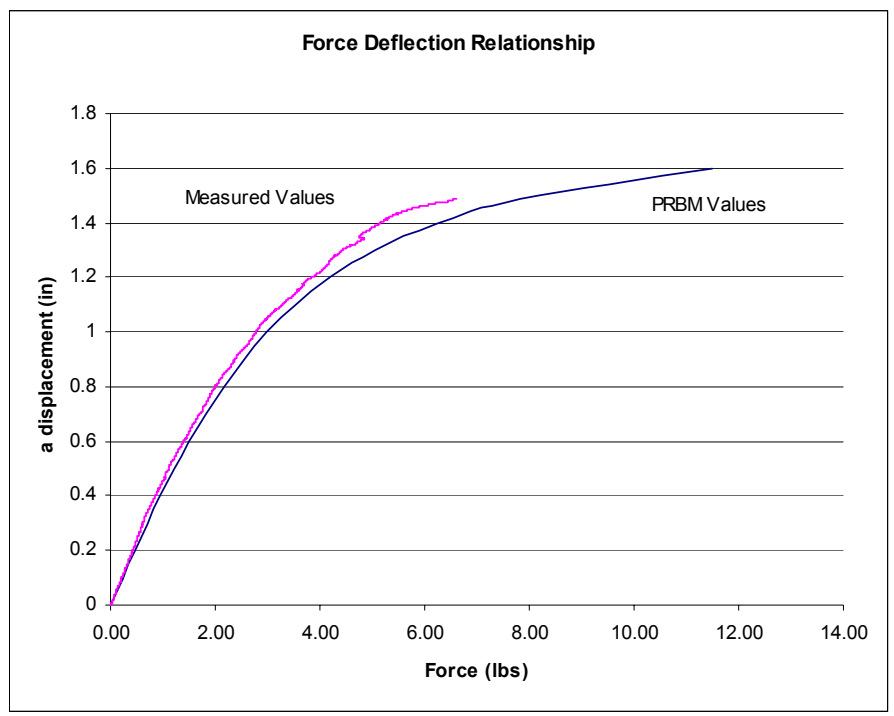

Figure 8. Measured values vs. PRBM calculated values for force and displacement

actuator was attached to an air bearing to allow for " $a$ " direction displacements. Potentiometers measured the actual displacements and the acquired data was sent directly to an integrated computer. A piezoelectric strain gage, attached at the displacement input, was used to acquire force data for all displacement values. The force data was collected by the computer. Figure 8 plots the force-deflection curve based on the PRBM equations and compares them to measured values from the prototype mechanism. Both Figure 7 and Figure 8 show that the PRBM accurately models the characteristics of this compliant mechanism.

The models described above were checked using a commercial finite element analysis program capable of nonlinear analysis 
(ANSYS) and by solving the non-linear deflections using elliptic integrals. The PRBM generated results that were within $98 \%$ of the results calculated by the finite element model and the elliptic integral solution and so the results from these other methods are not included in the figures.

The width of the compliant member can be changed to increase the force required for a given deflection without affecting the stress, as demonstrated in Equation (5) and Equation (6). The stress in the flexible member, $S$, is

$$
S=\frac{2 K_{\Theta} E a h \Theta}{l^{2} \cos \Theta}
$$

Fatigue analysis was also performed using the stresses found from the PRBM and by using the modified Goodman diagram. Table 4 lists the assumptions made in the analysis. Based on the assumptions stated, the fatigue life prediction for E-Glass is 450,000 cycles. Multiple samples of four and five layer E-Glass composites were tested to 110,000 cycles without failure. One cycle represents a full shift from the smallest sprocket to the largest sprocket and back to the smallest sprocket. The 110,000 cycles is equivalent to 30 cycles per day, 7 days a week for 10 years.

In conclusion, the PRBM allowed for the quick and accurate analysis of a simple but innovative compliant mechanism used for a commercial bicycle derailleur. The results from the PRBM match very closely to the actual tested values and to the values generated by traditional nonlinear analysis and FEA methods. The stresses from the PRBM were also used in fatigue life calculations. Currently a patent for the compliant derailleur design is pending and various derailleur manufacturers are considering it for use as a consumer product. The final two prototypes are shown in Figure 9 and Figure 10.

Table 4: Fatigue Calculation Information

\begin{tabular}{cc}
\hline Fatigue Assumptions & Value \\
\hline $\mathrm{S}_{\mathrm{ut}}$ for E-Glass & $3.4 \mathrm{GPa}(500,000 \mathrm{psi})$ \\
$\mathrm{S}_{\mathrm{e}}$ for E-Glass & $1.7 \mathrm{GPa}(250,000 \mathrm{psi})$ \\
Correction Factor $\mathrm{C}_{\text {surf }}$ & 0.9 \\
Correction Factor $\mathrm{C}_{\text {size }}$ & 1 \\
Correction Factor $\mathrm{C}_{\text {load }}$ & 1 \\
Correction Factor $\mathrm{C}_{\text {relaiblity }}$ & 0.75 \\
Correction Factor $\mathrm{C}_{\text {misc }}$ & 0.8 \\
$\mathrm{C}_{\mathrm{f}}$ & 0.9 \\
$\mathrm{~S}_{\mathrm{Max}}=2 \mathrm{~S}_{\mathrm{Alt}}$ & $1.1 \mathrm{GPa}(150,000 \mathrm{psi})$ \\
\hline
\end{tabular}

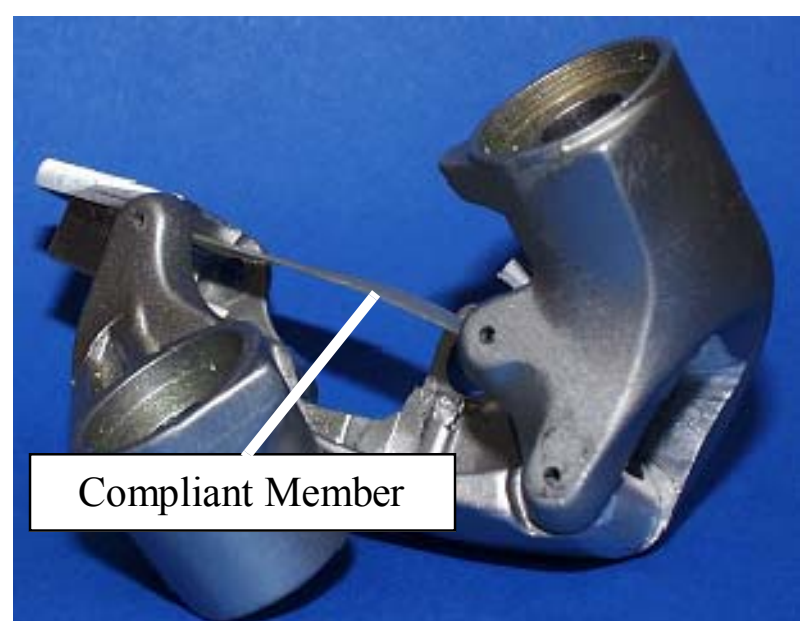

Figure 9. Final prototype representing small tooling changes to existing derailleurs to accommodate the compliant member

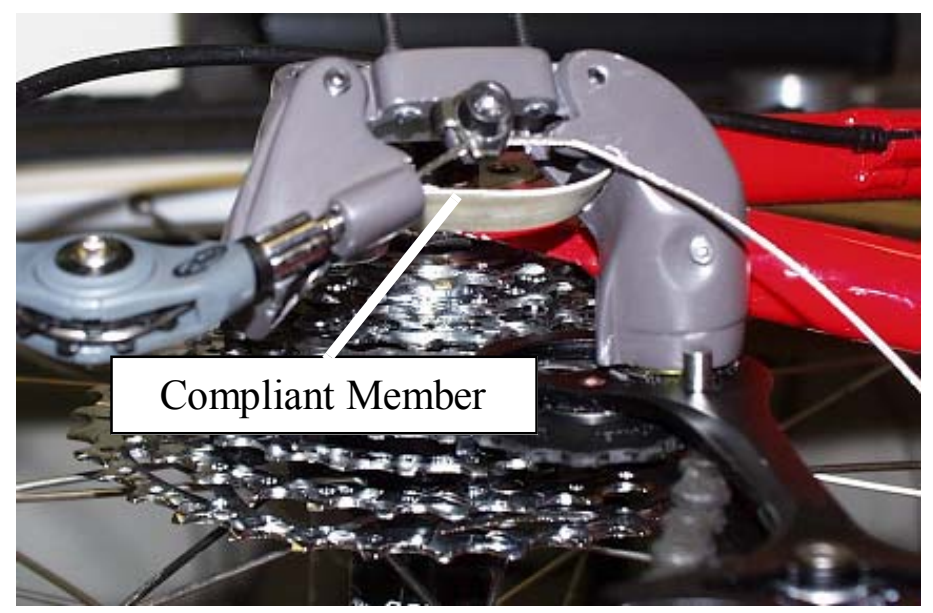

Figure 10. Final prototype for a fully re-tooled derailleur

Another case study, similar to the compliant derailleur, is the compliant parallel-motion bicycle brakes. This is the focus of the following section.

\section{CASE STUDY - COMPLIANT BICYCLE BRAKES}

Cantilever-type brakes have been commonly used on off-road bicycles because of their braking power and simplicity. When the rider actuates the brake handle, a cable causes the arms to rotate until the brake pads contact the rim of the wheel to provide a friction force for stopping. Cantilever brakes with elongated arms were introduced to provide increased mechanical advantage during actuation, resulting in a larger normal force at the pad for a given input force. The advantages of the elongatedarm cantilever brakes were further enhanced by the introduction of long-armed brakes that were configured as part of a four-bar parallelogram mechanism. The resulting motion caused the brake pads to move normal to the wheel rim, resulting in the 


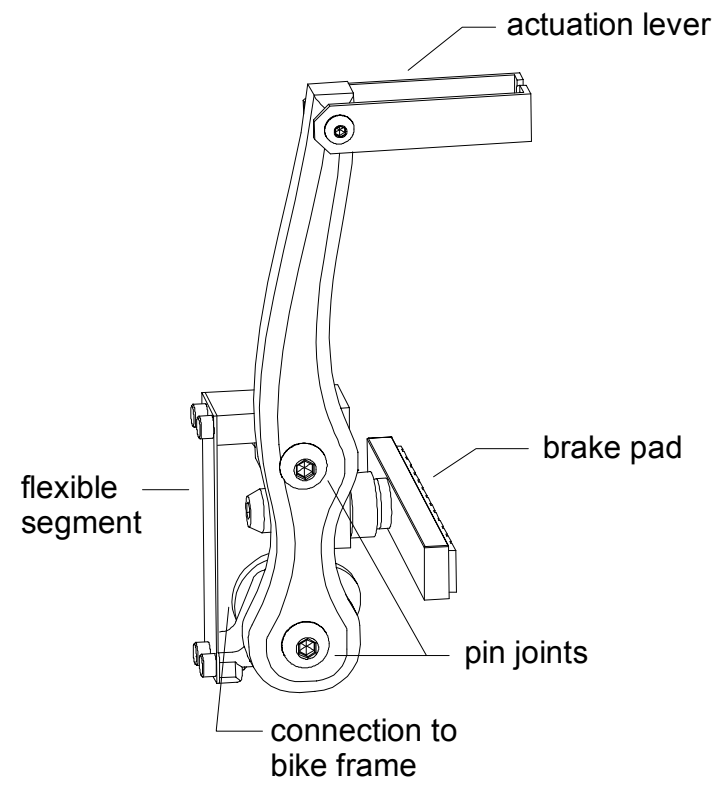

(a)

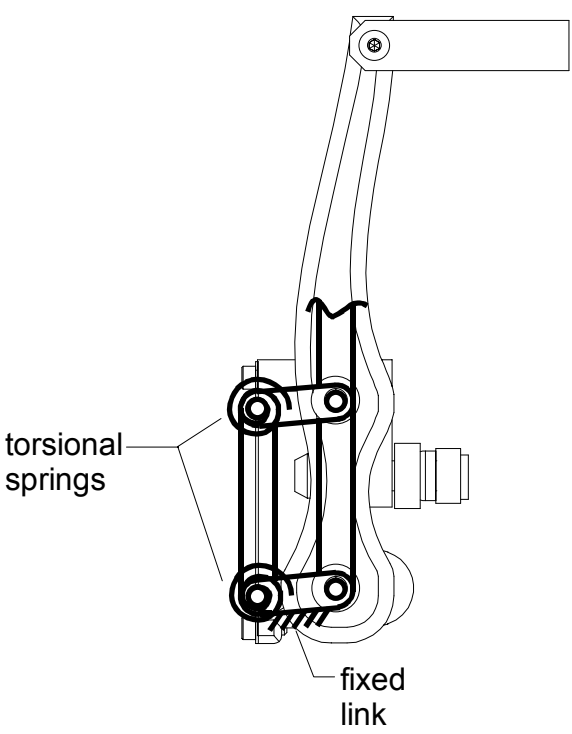

(b)

Figure 11. (a) Compliant bicycle brakes with brake pads that translate but do not rotate, and (b) the mechanism's pseudo-rigid-body model.

pads contacting the rim in a consistent manner. This helps eliminate uneven pad wear and reduces the need to adjust the pads to compensate for wear.

In spite of the advantages of parallel-motion brakes over simple single-arm brakes, the rigid-body, four-bar, parallelogram configuration suffers from several problems. The increased complexity of adding the pin joints increases cost and maintenance. Riders complain of undesirable motion between connecting parts, especially under heavy load conditions. Vibrations and noise, including those associated with the return spring, caused manufacturers to respond with special kits to deal with the problems.

Compliant parallel-motion brakes have been designed to exploit the advantages of parallel-motion brakes while reducing or eliminating the disadvantages associated with rigid-body configurations. As in the case of the compliant derailleur, the PRBM was critical in the development of the compliant parallelmotion brakes.

Again, type synthesis was used and the twenty-eight possible parallel-mechanism configurations were investigated through a series of analyses and prototypes until a configuration was selected. This configuration allows reduced part count compared to the rigid-body counterpart, integrates the return spring with the motion components, and can withstand large loads because they are carried by the rigid components.

A sketch of the compliant bicycle brakes is shown in Figure 11a. The PRBM for the brakes is a parallelogram linkage, as shown in Figure 11b. The force-displacement relationships need

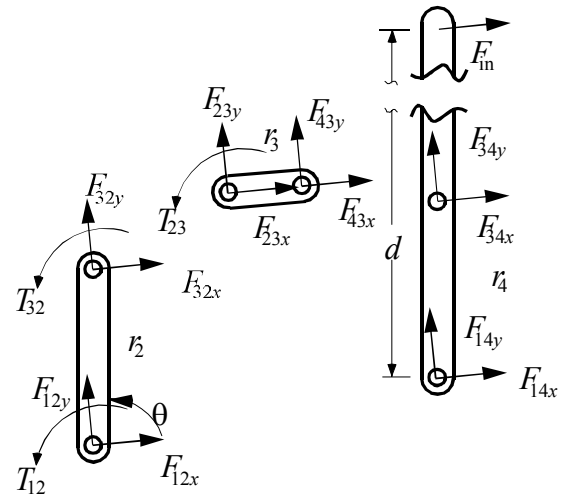

Figure 12. The free-body diagrams for the links of the compliant bicycle brakes.

to be understood so that the flexible beam can be designed in a manner that provides adequate spring force, but does not require too much input force. The free-body diagrams for each link are illustrated in Figure 12. The brake pad translates but does not rotate. The $x$-axis is chosen to be along link 3 such that $\theta_{3}=0$. For simplicity in the example, it is assumed that $F_{\text {in }}$ is in the $x$ direction. The equations of static equilibrium for link 2 are

$$
\begin{gathered}
F_{12 x}+F_{32 x}=0 \\
F_{12 y}+F_{32 y}=0 \\
T_{12}+T_{32}+F_{32 y} r_{2} \cos \theta_{2}-F_{32 x} r_{2} \sin \theta_{2}=0
\end{gathered}
$$


The equations for link 3 are

$$
\begin{aligned}
& F_{23 x}+F_{43 x}=0 \\
& F_{23 y}+F_{43 y}=0 \\
& T_{23}+r_{3} F_{43 y}=0
\end{aligned}
$$

and the equations associated with link 4 are

$$
\begin{gathered}
F_{34 x}+F_{14 x}+F_{\text {in }}=0 \\
F_{34 y}+F_{14 y}=0 \\
F_{34 y} r_{4} \cos \theta_{4}-F_{34 x} r_{4} \sin \theta_{4}-F_{\text {in }} d \sin \theta_{4}=0
\end{gathered}
$$

where $d$ is the distance from the ground pin of link 4 to the location of the input force.

The loads on two links connected at a pin joint have forces with equal magnitudes but act in opposite directions, or

$$
\begin{aligned}
& F_{32 x}=-F_{23 x} \\
& F_{32 y}=-F_{23 y} \\
& F_{43 x}=-F_{34 x} \\
& F_{43 y}=-F_{34 y}
\end{aligned}
$$

Combining the previous equations with the equilibrium equations results in

$$
F_{43 x}=-F_{34 x}=F_{32 x}=-F_{23 x}=-F_{12 x}
$$

and

$$
F_{43 y}=-F_{34 y}=F_{32 y}=-F_{23 y}=-F_{12 y}
$$

Because the pseudo-rigid-body model is a parallelogram mechanism, links 2 and 4 have the same rotation angle, $\theta$, or

$$
\theta_{2}=\theta_{4}=\theta
$$

The torques at the pin joints due to the springs are

$$
T_{12}=T_{32}=-T_{23}=-K\left(\theta-\theta_{0}\right)
$$

where $\theta$ is the angle of link 2 and 4 , and $\theta_{o}$ is the initial angular position of links 2 and 4 when the torsional springs are undeflected. The torsional spring constant, $K$, for the fixedguided flexible segment is (Howell, 2001)

$$
K=2 \gamma K_{\Theta} \frac{E I}{l}
$$

Equations (12) and (23) may be rearranged to obtain

$$
F_{43 y}=\frac{K\left(\theta-\theta_{o}\right)}{r_{3}}
$$

and equation (9) may be combined with equations (21) and (23) to solve for $F_{32 x}$ as

$$
F_{32 x}=\frac{K\left(\theta_{o}-\theta\right)\left(2+\frac{r_{2}}{r_{3}} \cos \theta\right)}{r_{2} \sin \theta}
$$

Combining equations (20) and (26) provides several of the reaction forces in the $x$ direction. The input force, $F_{\text {in }}$, is found by rearranging equations (15), (20), (21), (23), (25), and (26) to obtain

$$
F_{\text {in }}=\frac{2 K\left(\theta_{0}-\theta\right)}{d \sin \theta}
$$

It is evident from the derailleur example how the pseudo-rigidbody model equations can be extended to perform the stress and fatigue analysis.

For weight and strength reasons, prototypes were constructed using titanium for the flexible member. However, steel was used for the final production version of the brakes for cost and manufacturing reasons.

Tektro Brake Systems licensed the compliant parallel-motion brake technology from Brigham Young University and now mass produces and markets them. They are able to produce the parallel-motion brakes for a third of the manufacturing cost of their competitor's rigid-body parallel-motion brakes, while eliminating the components that were causing reliability problems in their competitor's design. Photos of mountain bike and BMX versions of the brake are shown in Figure 13a and Figure 13b, respectively.

\section{CONCLUSIONS}

The case studies presented in this paper have been used to demonstrate that the pseudo-rigid-body model (PRBM) can be used to quickly and accurately develop compliant mechanisms that are commercially viable. Specifically the PRBM was used to

1. Identify, analyze, and select an appropriate compliant configuration

2. Calculate the force-deflection relationships

3. Perform stress and fatigue analyses

4. Select materials appropriate for the given stress and fatigue loads, considering necessary force constraints 


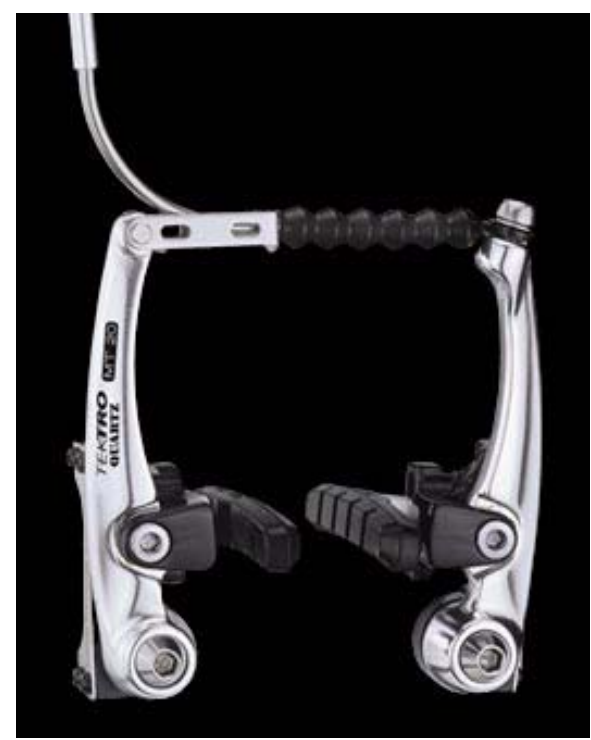

(a)

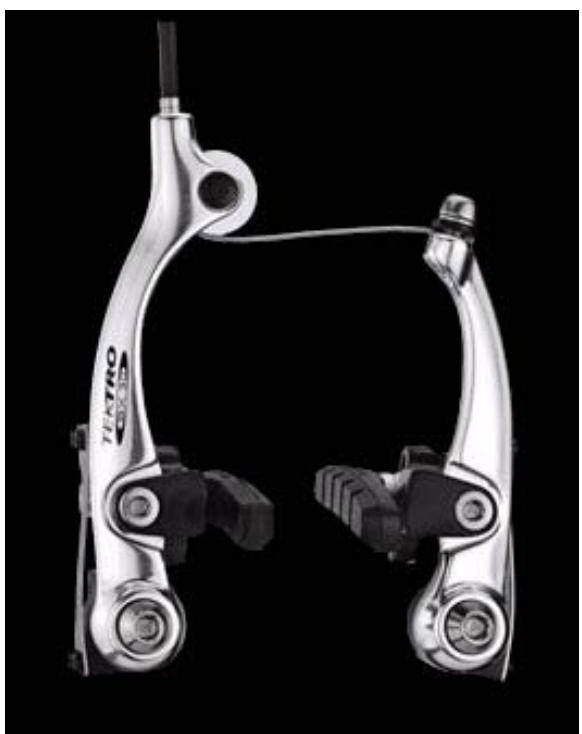

(b)

Figure 13: Commercially available compliant parallel-motion (a) mountain bike brakes (MT 20), and BMX brakes (BX 30), courtesy of Tektro Braking Systems.

5. Determine dimensions that meet constraints of fatigue life and return force

The implementation of compliant mechanism technology through the use of the PRBM can aid designers in developing compliant mechanisms that are innovative, have decreased weight and part-count and demonstrate increased precision. These benefits are illustrated in the two case studies presented in this paper. Prototypes of the compliant rear derailleur and compliant parallel-motion brakes have been successfully designed and tested. The compliant brakes have proven commercially viable as they are currently mass produced and the compliant derailleur has proven attractive to various derailleur manufactures as a decrease in weight is significant and minimal tooling changes may be made to existing derailleurs to accommodate the compliant design.

\section{ACKNOWLEDGEMENTS}

The authors would like to thank the Utah Center of Excellence for Compliant Mechanisms and the State of Utah for funding this project, the Mechanical Engineering Capstone Program at Brigham Young University, and the senior engineering students and graduate students that worked to develop these products. The support of the National Science Foundation, through grant NSF-9624574.

\section{REFERENCES}

Ananthasuresh, G.K., and Kota, S., 1995, "Designing Compliant Mechanisms," Mechanical Engineering, Vol 117, No. 11, pp. 9396.

Berglund, M.D., Magleby, S.P., and Howell, L.L., 2000, "Design Rules for Selecting and Designing Compliant Mechanisms for Rigid-Body Replacement Synthesis," Proceedings of the 2000 ASME Design Technical Conferences, DETC00/DAC-14225.

Derderian, J.M., Howell, L.L., Murphy, M.D., Lyon, S.M., and Pack, S.D., 1996, "Compliant Parallel-Guiding Mechanisms," Proceedings of the 1996 ASME Mechanisms Conference, 96DETC/MECH-1208.

Erdman, A.G., and Sandor, G.N., 1997, Mechanism Design: Analysis and Synthesis, Vol. 1, Third Edition, Prentice-Hall, Englewood Cliffs, NJ.

Frecker, M.I., "Design of Efficient Compliant Mechanisms from Ground Structure-Based Optimal Topologies," Proceedings of the 26th Design Automation Conference, 2000 ASME Design Engineering Technical Conferences, Baltimore, Maryland, DETC2000/MECH-14142.

Howell, L.L., Midha, A., and Norton, T.W., 1996, "Evaluation of Equivalent Spring Stiffness for Use in a Pseudo-Rigid-Body Model of Large-Deflection Compliant Mechanisms," ASME Journal of Mechanical Design, Vol. 118, pp 125-131.

Howell, L.L., 2001, Compliant Mechanisms, John Wiley and Sons, New York, NY. 
Murphy, M.D., Midha, A., and Howell, L.L., "The Topological Synthesis of Compliant Mechanisms," Mechanism and Machine Theory, Vol. 31, No. 2, pp. 185-199, 1996.

Pedersen, C.B.W., Buhl, T., Sigmund, O., 1999, “Topology Synthesis of Large-Displacement Compliant Mechanisms," Proceedings of the 25th Design Automation Conference, 1999 ASME Design Engineering Technical Conferences, Las Vegas, Nevada, DETC1999/DAC-8554. 